\title{
Generation and Analysis of a New Implicit Difference Scheme for the Korteweg-de Vries Equation
}

\author{
Yuri Blinkov ${ }^{1, \star}$, Vladimir Gerdt ${ }^{2, \star \star}$, and Konstantin Marinov ${ }^{3, \star \star \star}$ \\ ${ }^{1}$ Saratov State University, Astrakhanskaya Street 83, 410012 Saratov, Russia \\ ${ }^{2}$ Joint Institute for Nuclear Research, Joliot-Curie 6, 141980 Dubna, Russia \\ ${ }^{3}$ International University "Dubna", Universitetskaya 19, 141980 Dubna, Russia
}

\begin{abstract}
In this paper we apply our computer algebra based algorithmic approach to construct a new finite difference scheme for the two-parameter form of the Korteweg-de Vries equation. The approach combines the finite volume method, numerical integration and difference elimination. Being implicit, the obtained scheme is consistent and unconditionally stable. The modified equation for the scheme shows that its accuracy is of the second order in each of the mesh sizes. Using exact one-soliton solution, we compare the numerical behavior of the scheme with that of the other two schemes known in the literature and having the same order of accuracy. The comparison reveals numerical superiority of our scheme.
\end{abstract}

\section{Introduction}

In our previous paper [1] the general algorithmic approach suggested in [2] for generating difference schemes was worked out for evolution equations of the form

$$
u_{t}=\partial_{x}\left(P+a u_{m-1}\right), \quad u_{m}:=\partial_{x}^{m} u, \quad P \in \mathcal{R}, \quad \operatorname{ord}_{\partial_{x}}(P)<m-1, \quad m \geq 2, \quad a \in \mathbb{R},
$$

where $\mathcal{R}$ is the ordinary differential polynomial ring with undeterminate $u$ and derivative $\partial_{x}$. We denote the differential order of $P$ by $\operatorname{ord}_{\partial_{x}}(P)$. The (differential) coefficient field of $\mathcal{R}$ is assumed to be a field of rational functions in parameters (constants) the coefficients of which are rational numbers.

In the present paper we generate and study a new difference scheme for the two-parameter form of the Korteweg-de Vries (KdV) equation

$$
u_{t}+\left(P+\beta u_{x x}\right)_{x}=0, \quad P=\frac{\alpha}{2} u^{2}, \quad \alpha, \beta \in \mathbb{R}
$$

which belongs to the class (1). Eq. (2) was extensively studied in the literature both analytically and numerically (see monograph [3] and refereces therein). It is integrable by the inverse scattering transform method, possesses a rather wide class of exact solutions and infinitely many local conservation laws. Besides, various difference schemes have been derived for its numerical solution. The paper is organized as follows. In Sect. 2 we describe the generation of a new difference scheme by our method and prove its convergence. In Sect. 3 we compare the numerical behavior of the obtained scheme with

\footnotetext{
$\star$ e-mail: BlinkovUA@info.sgu.ru

$\star \star$ e-mail: gerdt@jinr.ru

$\star \star \star$ e-mail: marinov.kohctahtih@gmail.com
} 
that of two other schemes, see [3], having the same order of accuracy. The exact one-soliton solution of (2) is used as a benchmark.

\section{Generation of a difference scheme for the $\mathrm{KdV}$ equation}

To generate a difference scheme for Eq. (2) we follow [1,2] and use a regular grid with $t_{n+1}-t_{n}=\tau$, $x_{j+1}-x_{j}=h$. First, we convert Eq. (2) into the integral conservation law form

$$
u_{t}+\left(P+\beta u_{x x}\right)_{x}=0 \Longrightarrow \oint_{\Gamma}-\left(P+\beta u_{x x}\right) d t+u d x=0
$$

where $\Gamma$ is the following rectangular integration contour ("control volume")

and add to Eq. (3) the integral relations

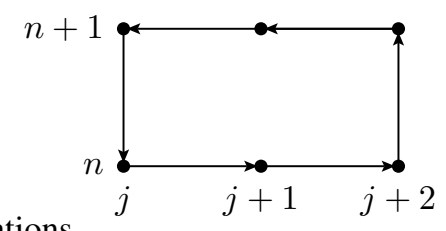

$$
\int_{x_{j}}^{x_{j+2}} u_{x x} d x=u_{x}\left(t, x_{j+2}\right)-u_{x}\left(t, x_{j}\right), \int_{x_{j}}^{x_{j+1}} u_{x} d x=u\left(t, x_{j+1}\right)-u\left(t, x_{j}\right) .
$$

Then, we evaluate the integrals (3) and (4) numerically. To evaluate (3), we use the trapezoidal rule for integration over $t$ and the midpoint rule for integration over $x$. The left integral in (4) is evaluated by the trapezoidal rule and the right integral by the midpoint rule. This results in a system of difference equations for the grid functions $u_{j}^{n},\left(u_{x}\right)_{j}^{n},\left(u_{x x}\right)_{j}^{n}$. The algebraic (difference) elimination of $\left(u_{x}\right)_{j}^{n}$ and $\left(u_{x x}\right)_{j}^{n}$ from this system yields a difference scheme for Eq. (2). Such elimination can be done by using MAPLE (see [1]).

The derived difference scheme

$$
\begin{gathered}
\frac{u_{j}^{n+1}-u_{j}^{n}}{\tau}+\frac{\alpha\left(\left(u^{2}\right)_{j+1}^{n+1}-\left(u^{2}\right)_{j-1}^{n+1}\right)+\alpha\left(\left(u^{2}\right)_{j+1}^{n}-\left(u^{2}\right)_{j-1}^{n}\right)}{8 h}+ \\
\frac{\beta\left(u_{j+2}^{n+1}-2 u_{j+1}^{n+1}+2 u_{j-1}^{n+1}-u_{j-2}^{n+1}\right)+\beta\left(u_{j+2}^{n}-2 u_{j+1}^{n}+2 u_{j-1}^{n}-u_{j-2}^{n}\right)}{4 h^{3}}=0
\end{gathered}
$$

can be solved by the simple iteration method (cf. [1]).

Using the library SyмPy [4] written in Рутном we computed the modified equation (or the first differential approximation [5]) of the scheme

$$
\begin{gathered}
u_{t}+\alpha u u_{1}+\beta u_{3}+h^{2}\left[\alpha\left(\frac{1}{6} u u_{3}+\frac{1}{2} u_{1} u_{2}\right)+\frac{\beta}{4} u_{5}\right]+\tau^{2}\left[\alpha^{3}\left(\frac{1}{12} u_{3}+\frac{3}{4} u^{2} u_{1} u_{2}+\frac{1}{2} u u_{1}^{3}\right)+\right. \\
\alpha^{2} \beta\left(\frac{1}{4} u^{2} u_{5}+\frac{7}{4} u u_{1} u_{4}+\frac{11}{4} u u_{2} u_{3}+\frac{9}{4} u_{1}^{2} u_{3}+\frac{11}{4} u_{1} u_{2}^{2}\right)+\alpha \beta^{2}\left(\frac{1}{4} u u_{7}+u_{1} u_{6}+\right. \\
\left.\left.\frac{9}{4} u_{2} u_{5}+\frac{7}{2} u_{3} u_{4}\right)+\frac{\beta^{3}}{12} u_{9}\right]+O\left(\tau^{4}, \tau^{2} h^{2}, h^{4}\right)=0, \quad u_{k}:=\partial_{x}^{k} u .
\end{gathered}
$$

The scheme (5) is implicit, and hence it is unconditionally stable. Eq. (6) shows the consistency of (5) with (2). Therefore, (5) is convergent (cf. [6]). 


\section{Numerical behavior of the generated scheme}

We compare the numerical behavior of scheme (5) with that of two other schemes from [3]. Similar to the scheme (5), these two schemes provide $O\left(\tau^{2}, h^{2}\right)$ approximation to Eq. (2).

Scheme I. The explicit scheme ([3], Eq. 1.80):

$$
u_{i}^{n+1}=u_{i}^{n-1}-\frac{\alpha \tau}{h} u_{i}^{n}\left(u_{i+1}^{n}-u_{i-1}^{n}\right)-\frac{\beta \tau}{h^{3}}\left(u_{i+2}^{n}-2 u_{i+1}^{n}+2 u_{i-1}^{n}-u_{i-2}^{n}\right)
$$

stable for

$$
\tau \leq \frac{2 h^{3}}{3 \sqrt{3} \beta} \cong 0.384 \frac{h^{3}}{\beta} \text {. }
$$

The modified equation reads

$$
\begin{aligned}
& u_{t}+\alpha u u_{1}+\beta u_{3}+h^{2}\left(\frac{\alpha}{6} u u_{3}+\frac{\beta}{4} u_{5}\right)-\tau^{2}\left[\alpha^{3}\left(\frac{1}{6} u^{3} u_{3}+\frac{3}{2} u^{2} u_{1} u_{2}+u u_{1}^{3}\right)+\alpha^{2} \beta\left(\frac{1}{2} u^{2} u_{5}+\frac{7}{2} u u_{1} u_{4}+\right.\right. \\
& \left.\left.\frac{11}{2} u u_{2} u_{3}+\frac{9}{2} u_{1}^{2} u_{3}+\frac{11}{2} u_{1} u_{2}^{2}\right)+\alpha \beta^{2}\left(\frac{1}{2} u u_{7}+2 u_{1} u_{6}+\frac{9}{2} u_{2} u_{5}+7 u_{3} u_{4}\right)+\frac{\beta^{3}}{6} u_{9}\right]+O\left(\tau^{4}, h^{4}\right)=0
\end{aligned}
$$

Scheme II. The implicit scheme ([3], Eq. 1.96):

$$
\begin{gathered}
\frac{u_{j}^{n+1}-u_{j}^{n}}{\tau}+\frac{\alpha}{4 h}\left[u_{j}^{n}\left(u_{j+1}^{n+1}-u_{j-1}^{n+1}\right)+u_{j}^{n+1}\left(u_{j+1}^{n}-u_{j-1}^{n}\right)\right]+ \\
\frac{\beta}{4 h^{3}}\left(u_{j+2}^{n+1}-2 u_{j+1}^{n+1}+2 u_{j-1}^{n+1}-u_{j-2}^{n+1}+u_{j+2}^{n}-2 u_{j}^{n+1}+2 u_{j-1}^{n}-u_{j-2}^{n}\right)=0
\end{gathered}
$$

the modified equation of which is given by

$$
\begin{aligned}
u_{t}+ & \alpha u u_{1}+\beta u_{3}+h^{2}\left(\frac{\alpha}{6} u u_{3}+\frac{\beta}{4} u_{5}\right)+\tau^{2}\left[\alpha^{3}\left(\frac{1}{12} u^{3} u_{3}+\frac{1}{4} u^{2} u_{1} u_{2}\right)+\frac{\alpha^{2} \beta}{4}\left(u^{2} u_{5}+5 u u_{1} u_{4}+7 u_{1}^{2} u_{3}\right.\right. \\
& \left.\left.+9 u u_{2} u_{3}+11 u_{1} u_{2}^{2}\right)+\alpha \beta^{2}\left(\frac{1}{4} u u_{7}+u_{1} u_{6}+\frac{9}{4} u_{2} u_{5}+3 u_{3} u_{4}\right)+\frac{\beta^{3}}{12} u_{9}\right]+O\left(\tau^{4}, \tau^{2} h^{2}, h^{4}\right)=0 .
\end{aligned}
$$

We performed numerical comparison of the difference schemes (5), (7) and (9) by using the Python-based library ScIPy [7]. As a benchmark, we considered the initial value problem $u(0, x)=$ $u_{\text {exact }}(0, x)$ for the exact one-soliton solution

$$
u_{\text {exact }}(t, x)=\frac{2 k_{1}^{2}}{\cosh \left(k_{1}\left(x-4 k_{1}^{2} t\right)\right)^{2}}
$$

to the $\mathrm{KdV}$ equation (2) with $\alpha=6, \beta=1$ and $k_{1}=0.4$. In so doing, for all schemes we fixed the spacial mesh step $h=0.25$. Then, for the explicit scheme (7) we fixed $\tau=0.37 h^{3} / \beta$, and for the implicit schemes (5), (9) we considered the following two values of $\tau: \tau=0.1 h$ and $\tau=h$. For these values of the mesh sizes we constructed a numerical solution in the interval $-50 \leq x \leq 50$ with periodic boundary conditions (cf. [3], p.49). The numerical accuracy was estimated by the Frobenius norm

$$
\left\|u-u_{\text {exact }}\right\|_{F}=: \sqrt{\sum_{n} \sum_{j}\left|u_{j}^{n}-u_{\text {exact }}\left(t_{n}, x_{j}\right)\right|^{2}} .
$$

The time dynamics of (12) for the schemes (5), (7) and (9) shown below (left pictures) demonstrates the superiority of our scheme (5) over the two other schemes. It is confirmed by the numerical values of the left-hand sides of the modified equations (6), (8) and (10) for the one-soliton solution (11) at $t=0$ (right pictures) $\tau$ and the equality of the terms of order $h^{2}$ in Eq. (8) and Eq. (10). 

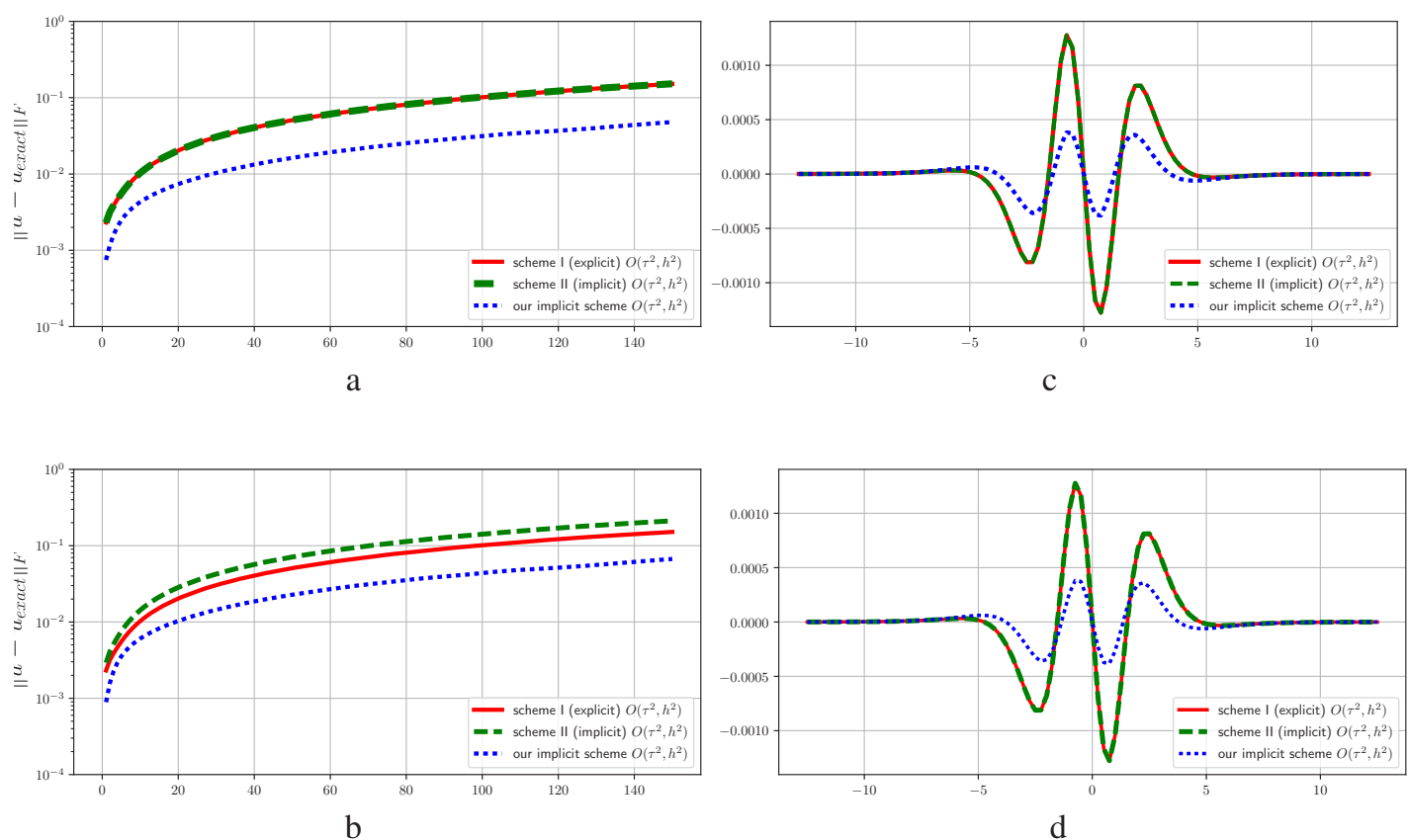

Figure 1. The time dynamics of descrepancy (12) for with $\tau=h / 10$ (a) and $\tau=h$ (b) for the implicit schemes, and the values (c) and (d) of the left-hand sides of the modified equations for these values of $\tau$, respectively.

\section{Conclusion}

By applying the modern methods of computer algebra related to differential and difference algebra, we generated and analysed the new implicit second order finite difference scheme (5) for the classical $\mathrm{KdV}$ equation. The symbolic analysis based on the construction of modified equations combined with numerical experiments demonstrated superiority of the scheme over the two other second order schemes known in the literature.

\section{Acknowledgements}

The work is supported in part by the Russian Foundation for Basic Research (grant 16-01-00080).

\section{References}

[1] V.P. Gerdt, Yu.A. Blinkov, and K.B. Marinov, Program. Comput. Soft. 43 (2), 84-89 (2017)

[2] Yu.A. Blinkov, V.P. Gerdt, and V.V. Mozzhilkin, SIGMA 2 (051) (2006) arXiv:math.RA/0605334

[3] V.Yu. Belashov and S.V. Vladimirov, Solitary Waves in Dispersive Complex Media. Theory. Simulation · Applications (Springer-Verlag, Berlin, 2005) 292 pp.

[4] http://www . sympy . org/en/

[5] Y.I. Shokin, The Method of Differential Approximation (Springer-Verlag, Berlin, 1983)

[6] E.E. Rosinger, Nonlinear Equivalence, Reduction of PDEs to ODEs and Fast Convergent Numerical Methods (Pitman, Boston, 1982)

[7] http://scipy.org 